\title{
Hardware-Accelerated Cross-Architecture Full-System Virtualization
}

\author{
TOM SPINK, HARRY WAGSTAFF, and BJÖRN FRANKE, University of Edinburgh, UK
}

\begin{abstract}
Hardware virtualization solutions provide users with benefits ranging from application isolation through server consolidation to improved disaster recovery and faster server provisioning. While hardware assistance for virtualization is supported by all major processor architectures, including Intel, ARM, PowerPC, and MIPS, these extensions are targeted at virtualization of the same architecture, for example, an x86 guest on an x86 host system. Existing techniques for cross-architecture virtualization, for example, an ARM guest on an x86 host, still incur a substantial overhead for CPU, memory, and I/O virtualization due to the necessity for software emulation of these mismatched system components. In this article, we present a new hardwareaccelerated hypervisor called CAPTIVE, employing a range of novel techniques that exploit existing hardware virtualization extensions for improving the performance of full-system cross-platform virtualization. We illustrate how (1) guest memory management unit (MMU) events and operations can be mapped onto host memory virtualization extensions, eliminating the need for costly software MMU emulation, (2) a block-based dynamic binary translation engine inside the virtual machine can improve CPU virtualization performance, (3) memory-mapped guest I/O can be efficiently translated to fast I/O specific calls to emulated devices, and (4) the cost for asynchronous guest interrupts can be reduced. For an ARM-based Linux guest system running on an x86 host with Intel VT support, we demonstrate application performance levels, based on

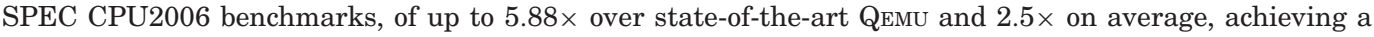
guest dynamic instruction throughput of up to 1280 MIPS (million instructions per second) and 915.52 MIPS, on average.
\end{abstract}

CCS Concepts: • Computing methodologies $\rightarrow$ Simulation tools; $\bullet$ Hardware $\rightarrow$ Simulation and emulation;

Additional Key Words and Phrases: Virtualization

ACM Reference Format:

Tom Spink, Harry Wagstaff, and Björn Franke. 2016. Hardware-accelerated cross-architecture full-system virtualization. ACM Trans. Archit. Code Optim. 13, 4, Article 36 (October 2016), 25 pages.

DOI: http://dx.doi.org/10.1145/2996798

\section{INTRODUCTION}

Virtualization technology enables workload consolidation where multiple applications are deployed onto Virtual Machines (VMs), which then run on a single, more-powerful host machine. While virtualization may introduce some runtime overhead, processor manufacturers have developed support in hardware in the form of instruction set extensions (ISEs) to make their respective architectures efficiently and fully virtualizable, for example, Intel VT or ARM Virtualization Extensions. They have also created additional mechanisms to allow I/O virtualization with less software overhead.

New paper, not an extension of a conference paper.

Authors' addresses: T. Spink, IF-1.47, Informatics Forum, 10 Crichton Street, Edinburgh, EH8 9AL; email: tspink@inf.ed.ac.uk; H. Wagstaff, IF-3.38, Informatics Forum, 10 Crichton Street, Edinburgh, EH8 9AL; email: hwagstaf@inf.ed.ac.uk; B. Franke, IF-1.04, Informatics Forum, 10 Crichton Street, Edinburgh, EH8 9AL; email: bfranke@inf.ed.ac.uk.

Permission to make digital or hard copies of part or all of this work for personal or classroom use is granted without fee provided that copies are not made or distributed for profit or commercial advantage and that copies show this notice on the first page or initial screen of a display along with the full citation. Copyrights for components of this work owned by others than ACM must be honored. Abstracting with credit is permitted. To copy otherwise, to republish, to post on servers, to redistribute to lists, or to use any component of this work in other works requires prior specific permission and/or a fee. Permissions may be requested from Publications Dept., ACM, Inc., 2 Penn Plaza, Suite 701, New York, NY 10121-0701 USA, fax +1 (212) 869-0481, or permissions@acm.org.

(c) 2016 ACM 1544-3566/2016/10-ART36 $\$ 15.00$

DOI: http://dx.doi.org/10.1145/2996798 
However, these virtualization extensions are geared towards same-architecture virtualization, where both the guest VM and the physical host machine share the same architecture. For cross-architecture virtualization, where guest VM and host architectures differ, translation between instruction set architecture (ISAs), emulation of the guest system's memory management unit (MMU), interrupt handling, and I/O devices are typically implemented entirely in software, resulting in a substantial performance loss. For example, in full-system mode the gem5 architectural simulator [Binkert et al. 2011] takes about 30 minutes to boot into Linux on a current, mid-range host machine. While this performance level is sufficient for some computer architecture research, it is far too slow for any practical applications. QEMU [Bellard 2005], a popular cross-architecture full-system virtualizer using dynamic binary translation (DBT), is substantially faster but still suffers an up to $20 \times$ slowdown [Jones and Topham 2009] compared to native execution on the host. ${ }^{1}$

While same-architecture virtualization has become ubiquitous, there are fewer, but nonetheless important, applications for cross-architecture virtualization, for example, Android software development using the QEMU-based Android Emulator shipped with the Android Studio [Gerber and Craig 2015], which provides a full-system ARM environment for software developers using an x86 host machine; building ARM Docker [Merkel 2014] containers on x86 machines; providing fast-forwarding in samplingbased simulators [Sandberg et al. 2015]; or early-stage software development without a hardware target [Ceng et al. 2009]. All of these applications critically depend on fast cross-architecture virtualization due to unavailability or deliberate absence of a hardware platform supporting the chosen target ISA.

In this article, we develop a novel approach for speeding up cross-architecture virtualization and implement these ideas in a new cross-architecture hypervisor called CAPTIVE. The key idea is to eliminate performance bottlenecks by exploiting the existing virtualization hardware extensions originally devised for same-architecture virtualization. We target four distinct cross-architecture virtualization challenges and make the following contributions:

(1) We show how virtual-to-physical address translation can be accelerated through the use of virtualization extensions by mapping behavior of the guest MMU onto corresponding behavior of the host MMU—despite substantial differences between the two MMUs.

(2) We present a DBT system for the translation from the guest to host ISA, where a fast, block-based just-in-time (JIT) compiler that lives inside the native virtual machine compiles guest basic blocks to host native code.

(3) We develop an efficient mechanism to emulate the guest's memory mapped I/O devices by exploiting the MMU to detect device accesses.

(4) Finally, we devise an interrupt handling scheme, which correctly honors the guest's instruction boundaries, even if one guest instruction is mapped onto several host instructions, thus implementing precise, yet efficient, guest interrupts.

\subsection{Motivating Example}

It has been well established [Magnusson and Werner 1994; Chang et al. 2014; Wang et al. 2015; Hong et al. 2015] that emulation of a guest MMU is one of the most time-consuming parts of cross-architecture virtualization; therefore, in this motivating example, we will focus on the memory address translation process required for virtualization. For this, consider the diagram in Figure 1, which shows the percentage

\footnotetext{
${ }^{1}$ Native execution of a binary suitably compiled to the host ISA from the same sources, which have been used to build the binary for the guest's ISA.
} 


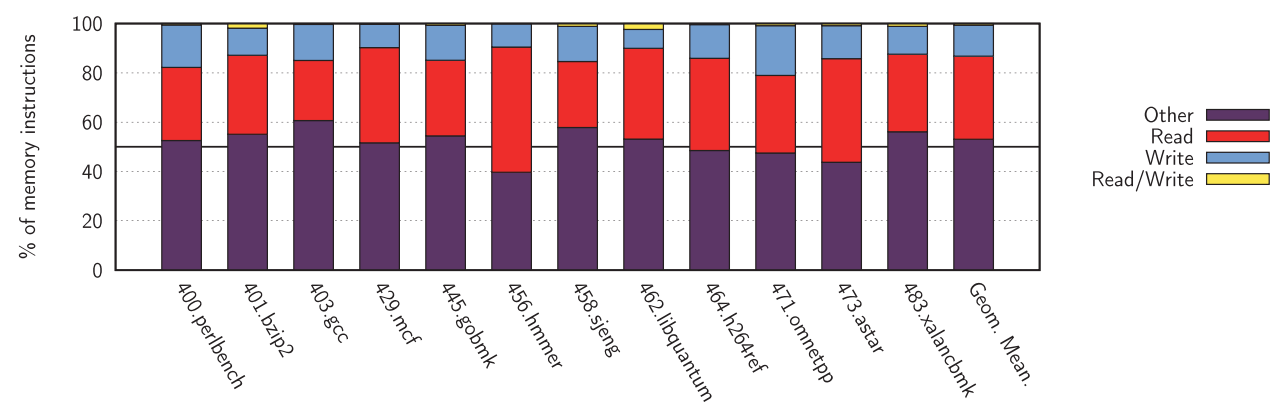

Fig. 1. Distribution of operations in the SPEC CPU2006 integer benchmarks. On average, around $50 \%$ of all instructions executed perform memory operations (read, write, or both), which require expensive virtualto-physical address translation using a software MMU.

of memory operations w.r.t. the total number of executed instructions in the SPEC CPU2006 integer benchmarks. About 50\% of the instructions in these benchmarks perform memory accesses. This means that if we are running these benchmarks in a virtualized ARM guest environment on an x86 host, then, on average, every other instruction demands an expensive virtual-to-physical memory translation using a virtualized ARM MMU, typically implemented in software. If we succeed in speeding up these address translations, then we will eliminate one of the most severe crossarchitecture virtualization performance bottlenecks.

In a 32-bit ARMv7-A system with an ARMv7-A MMU, there are at most two levels of page tables representing a virtual memory area. To translate a virtual address into its corresponding physical address, the first-level page table (an L1 page table), pointed to by the TTBR register, is indexed by bits 20-31 of the virtual address and the entry interrogated to determine if the mapping is to a section (a $1 \mathrm{MB}$ contiguous chunk of memory) or a small page (a $4 \mathrm{kB}$ contiguous chunk of memory). If the page table entry indicates a section, then the base address points to the physical base address of the memory. If the page table entry indicates a small page, then the base address points to the physical base address of a second-level page table (an L2 page table). The L2 page table is indexed by bits 12-19 of the virtual address, and the base address in the L2 page table entry points to the physical base address of the page corresponding to the mapping. A page table entry in the L1 (if pointing to a section) or L2 page table in addition to the base address pointer contains flags that indicate the access permissions of the page, for example, whether the page is readable and writable, and if it is accessible while executing in the user privilege level.

In a 64 -bit x 86 system, there are four levels of page tables that represent a 48-bit virtual address space. Pointers are always 64 -bit wide but can only access 48 bits of virtual address space. Virtual addresses must be in canonical form, where bits 48-63 of the address are copies of bit 47. Any memory access to a non-canonical address will result in a general protection fault. Address translation operates in a similar fashion to ARM, with each level of page table being traversed to translate a 64-bit virtual address into a 64-bit physical address, subject to permissions that can be applied at any level of the page table-with the higher-level permissions taking precedence over the lower levels.

To avoid a costly page table walk for every memory access, both architectures employ a translation lookaside buffer (TLB), which caches the result of a previous hardware translation. If the page tables are modified, or the pointer to the top-level page table changed, then the TLB must be flushed.

From this description, it should be clear that the structure of the ARM and x86 MMUs substantially differ, yet, fundamentally, they both provide a mechanism for the 
translation of virtual addresses to physical addresses with access permission checking. In this article, we propose to exploit this hardware address translation capability and show how to map the behavior of an ARM MMU onto a virtualized Intel MMU. By intercepting ARM TLB invalidations and maintaining entries in the x86 page table that represent entries in the ARM page table, we can accelerate address translations. Instead of using a slow software implementation of the ARM MMU, guest address translations are redirected to the fast, host virtualized Intel MMU, which our system keeps consistent with the guest's ARMv7-A MMU. Using existing extensions originally devised for same-architecture virtualization, we are able to speed up critical cross-architecture address translations over a purely software-based MMU implementation.

\subsection{Overview}

The remainder of this article is structured as follows. In Section 2 we provide background information on MMU virtualization in full-system simulators, Intel virtualization technology (Intel VT), and the kernel-based virtual machine (KVM) framework, on which our work relies. We then present our novel cross-architecture virtualization techniques in Section 3 and our results in Section 4. We discuss related work in Section 5, before we summarize and conclude in Section 6.

\section{BACKGROUND}

While this article is largely self-contained, we assume a certain level of familiarity with the Intel VT extensions and also KVM. In this section, we briefly introduce these technologies before describing our virtualization infrastructure in the next section.

\subsection{Intel VT}

Intel VT [Intel 2016] is a set of hardware extensions introduced by Intel to provide support for virtualization of an x86 processor. It provides new instructions for setting up and managing these virtual machines, transitioning between hypervisor and virtualized execution, and support for virtualizing the guest MMU with extended page tables (EPT). EPT provides an extra level of page tables that are walked by hardware when a virtual memory address that is not present in the TLB is encountered while running in the virtual machine. CAPTIVE utilizes Intel VT extensions by creating a virtual machine with the KVM infrastructure provided by the Linux kernel.

\subsection{KVM}

Hardware-accelerated virtualization is well supported by multiple processor vendors, such as Intel with Intel VT and AMD with AMD-V. While these extensions produce the same effect, that is, they create an abstract computing platform on which to run unmodified operating systems, they are implemented and accessed completely differently. KVM [2016] is a virtual machine monitor implemented as part of the Linux kernel, which can utilize any supported hardware virtualization on any platform. Its job is to abstract the details of the virtualization extensions and to provide a generic interface for creating and managing a virtualization environment. KVM fully supports virtualization extensions such as Intel EPT or AMD Rapid Virtualization Indexing (RVI), which is used to accelerate a virtualized MMU. KVM itself is only an interface to hardware virtualization extensions; it will not work in the absence of these.

KVM was developed in tandem with QEMU, but does not depend on QEMU-it is an independent technology that is part of the standard Linux kernel.

In CAPTIVE, we utilize KVM to create a virtual machine backed by Intel VT or AMD-V in a platform-agnostic way. The use of KVM, and, consequently, the use of Intel VT, is described in more detail in Section 3.1. 


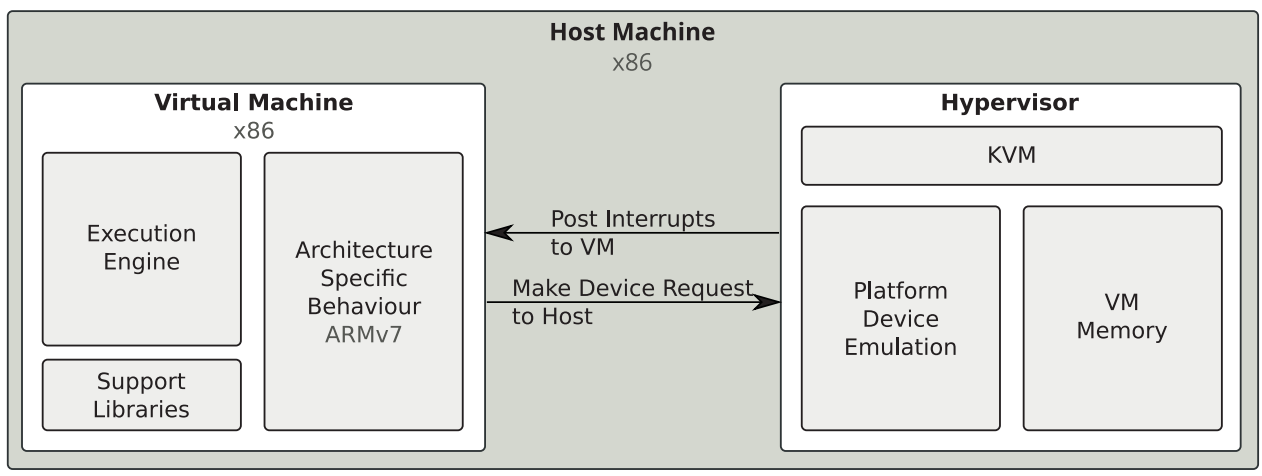

Fig. 2. A high-level overview of the virtualization infrastructure. The CAPTIVE hypervisor runs in the operating system (Linux) of the host machine and creates a virtual machine that is the same architecture as the host (x86). Inside this virtual machine, the CAPTIVE execution engine virtualises a guest platform (ARMv7) by mapping the behaviour of the guest to the behaviour of the host.

\section{VIRTUALIZATION INFRASTRUCTURE}

Our virtualization infrastructure consists of three main components:

(1) A hypervisor component, which runs on the host machine and uses KVM to control the host's hardware virtualization extensions.

(2) An execution engine component, which runs inside a normal virtual machine on the host.

(3) An architectural implementation, which defines the behavior of the architecture being virtualized.

For the rest of this article, we will be assuming that we are virtualizing an ARMv7A guest architecture (modelling a RealView Platform Baseboard for Cortex-A8 [ARM 2011b]), on a standard x86-64 host machine with Intel VT virtualization extensions.

Due to the multi-layer nature of this system, it is important to define some terminology at this point to establish exactly what terms will describe what aspects of the system.

Definition 3.1 (Host). The host machine is the system on which we will be running the virtualized platform. This article will use an x86-64 machine as the host machine.

Definition 3.2 (Guest). The guest machine is the target platform that we wish to virtualize. This article will use an ARMv7-A platform as the guest machine.

Definition 3.3 (Native Virtual Machine (VM)). The hardware extensions provided by the host machine naturally provide a same-architecture virtual machine, for example, using QEMU with KVM on x86 would result in an x86 virtual machine. In our case, the Native Virtual Machine (VM) refers to the virtual machine provided by these hardware extensions, to which we utilize in our infrastructure. Therefore, in this article, the Native VM is of the same architecture (x86-64) as the host.

Definition 3.4 (Hypervisor). The hypervisor is the software that runs on the host machine (within the host operating system) and is responsible for providing support to the Native VM, along with software implementations of guest platform devices (e.g., a timer device or an interrupt controller).

Definition 3.5 (Execution Engine). The execution engine is a bare-metal application that runs inside the Native VM (without an operating system) and provides the CPU virtualization necessary to run the cross-ISA guest instructions. It maintains 
the state of the guest platform being emulated and executes platform-specific behavior, such as what happens when an MMU fault occurs or an external interrupt is signalled.

\subsection{Overview}

This section will give an overview of the operation of CAPTIVE. It will continue to use the example of an x86-64 host machine, and an ARMv7-A guest machine, implementing the RealView Platform Baseboard Cortex-A8 [ARM 2011b]. This example leads to the following assumptions:

(1) We are virtualizing a 32-bit guest system on a 64-bit host system.

(2) We depend on KVM as a framework to provide access to Intel VT virtualization extensions.

(3) We utilize (but do not depend on) the address-space identifier (ASID) feature of the guest operating system to accelerate context switches.

(4) We exploit the fact that ARM page tables have similar access permissions to x86 page tables, enabling us to efficiently map MMU behavior.

Our system starts by instantiating a native virtual machine on the host, using the KVM framework. KVM is the interface to the Intel VT virtualization extensions and starts by initializing the required structures that represent a virtual machine on an Intel processor (e.g., creating the VMCS structure and issuing the VMXON instruction). Then, a single virtual x86-64 CPU is requested from KVM that will ultimately represent the virtual ARM processor.

This native VM is configured with physical memory that represents the physical memory provided by the guest platform-in this case, 2GB of physical memory is installed. An additional block of physical memory is also installed that contains the execution engine binary and heap space for memory allocations and the translated code cache. The guest kernel (an ARMv7-A Linux kernel) is written into the guest physical memory at the correct location (as specified by the platform boot protocol). Finally, the virtual x86-64 CPU is configured to start up in 64-bit mode (by manipulating the virtual CPU state structure) and with the entry-point of the execution engine. The native VM is then started, and control is transferred to the execution engine running inside.

Once inside the native VM, we have full control of a bare-metal x86-64 machine, the execution engine is essentially an x86-64 kernel, with full privileged access to this virtual machine. We configure the virtual memory of the native VM in such a way that the lower $4 \mathrm{~GB}$ portion represents either a one-to-one mapping of guest physical memory (if the guest MMU is turned off) or the actual virtual memory mapping of the guest machine (detailed in Section 3.3). The execution engine itself resides in the high portion of virtual memory, and certain other virtual memory areas are mapped to the heap and stack.

When first started, the execution engine performs some platform initialization of the native VM, such as setting up the native (x86) page tables and the interrupt descriptor table, and eventually begins executing guest ARM code. We have already populated guest physical memory with the guest kernel we are about to execute, so execution begins from this entry point, using JIT compilation of the guest ARM instructions to native x86-64 instructions (detailed in Section 3.2).

Any access by guest instructions to the memory of the guest machine is performed with a normal memory access, without having to translate/transform any virtual memory addresses - they are simply made to the address to which they would be made if running on a non-virtualized ARM system.

The guest platform we are virtualizing is a 32-bit platform, and so any memory access can only be in the $0-4 \mathrm{~GB}\left(2^{32}\right)$ range of lower virtual memory. Virtualization of 
a 64-bit platform is outside the scope of this article but will require an extra layer of software indirection and is planned for future work. When an access to a particular address occurs for the first time, a page fault is generated and handled by installing a mapping of the corresponding virtual page to guest physical memory (subject to the operation of the ARM guest MMU).

External interrupts generated by devices (such as a timer device) are propagated as real interrupts into the guest system, which causes a flag to be set to indicate that native code should stop executing at the next safe point. At a minimum, a safe point is an instruction boundary, but we insert safe points at guest basic block boundaries to improve performance.

\subsection{CPU Virtualization}

Same-architecture virtualization is easily supported by modern processors that include hardware support for virtualization. Technologies such as Intel VT and AMD-V allow guest code to run directly on the host processor, without modification or instrumentation for maximum performance. Certain privileged operations (such as changes to control registers and TLB invalidations) are trapped by the host CPU and handled by a hypervisor (for example, KVM).

This virtualization is trivial in the same-architecture case, because both the guest and the host have the same ISA and are therefore binary compatible. However, this presents a problem for cross-architecture CPU virtualization, as the ISAs different and are completely incompatible.

In a traditional full-system simulator, techniques such as interpretation or DBT are used to virtualize the guest ISA on the host ISA, the former being straightforward to implement, and the latter being recognized as one of the fastest ways [Ung and Cifuentes 2000; Ebcioglu et al. 2001; Bellard 2005] to emulate guest instructions on a host machine. Emulation of guest instructions is a necessity for cross-architecture virtualization, and techniques for doing so have been well studied and presented in DBT improvement articles such as Kumar et al. [2005] and Guha et al. [2010].

Our approach to instruction emulation is based on a basic block JIT compiler engine, which takes guest basic blocks discovered at runtime and compiles them into corresponding host basic blocks. Block compilation is synchronous to the execution of the guest system and occurs on-demand when a translation for a particular guest basic block is not available. Generated host code is stored in a code cache for later use. This is similar to the approach taken by QEMU, except for two important differences: (1) we generate code independent of the virtual address of the guest basic block and (2) the JIT compiler in CAPTIVE lives inside the native virtual machine as part of the execution engine.

QEMU has implemented an advanced caching strategy that initially uses a fast firstlevel cache indexed by virtual address to look up the code associated with a guest basic block, which is invalidated when page mappings change. As basic blocks are translated for specific virtual addresses, the virtual PC may be constant-folded into the translations. However, the translation cannot be re-used if the same physical address is mapped to different virtual addresses. To handle this situation, when a miss occurs in the first-level cache, a second-level cache that is indexed by virtual PC, physical PC, and memory access flags is consulted. If this cache misses, then the guest basic block is translated. This results in a guest basic block being translated for each distinct virtual address mapping. In contrast, CAPTIVE always indexes the code cache by physical PC, and translates code in a way that is independent of its virtual address, meaning we can re-use the same translation for multiple virtual addresses.

The JIT compiler is generated from a high-level instruction description, where instruction formats are defined in an ArchC-like domain-specific language (DSL) 
Listing 1: High-level instruction format description.

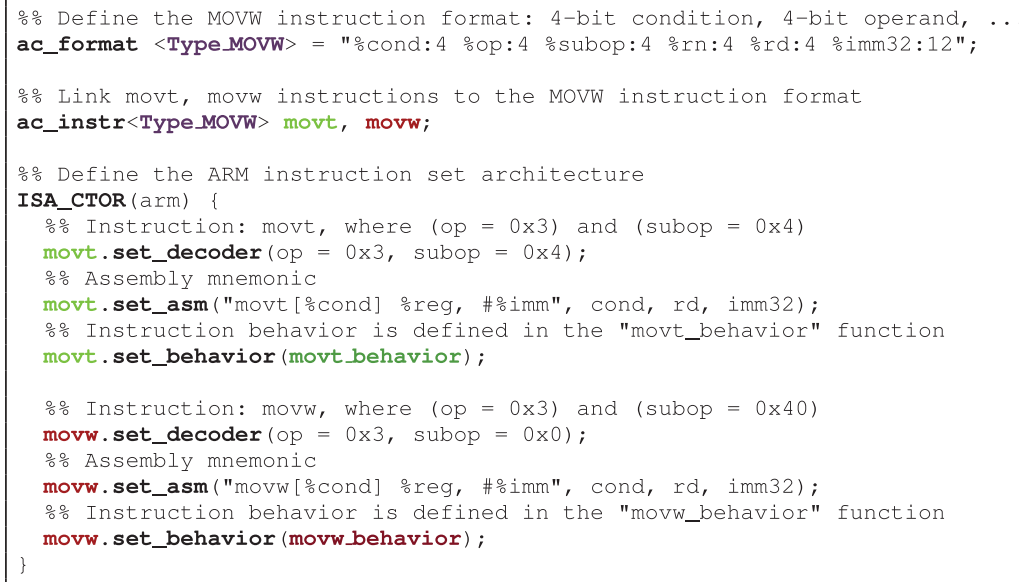

Listing 2: Semantic description of the behavior of the movt and movw instructions.

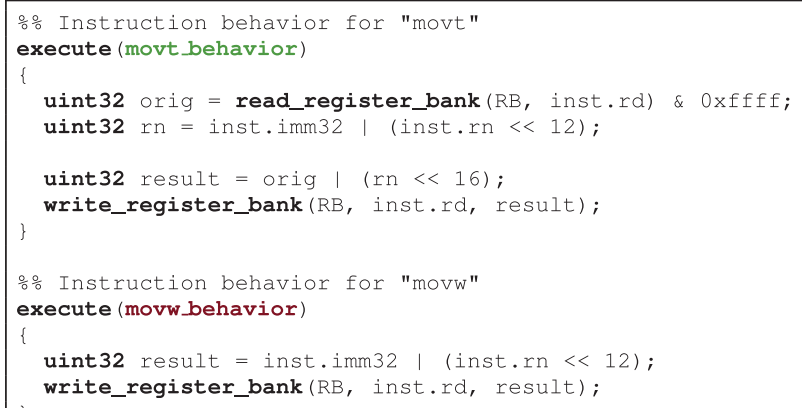

Fig. 3. Instruction formats and semantics are specified using an ArchC-like high-level architecture description language. Example showing the specification of formats (in Listing 1) and semantics (in Listing 2) of the movt and movw instructions, respectively.

[Azevedo et al. 2005], and the semantic behaviors are specified in a C-like DSL. In an offline pass, this architecture definition is parsed, and a high-speed instruction decoder and instruction intermediate representation (IR) generator are produced, which are then used by the execution engine to compile guest code.

In Figure 3, Listing 1 shows an extract of an instruction-format description from our ARMv7-A model, which is used to generate an instruction decoder. Line 2 contains a bit-level representation of the instruction format, and line 5 declares two instructions (movt and movw) that conform to this pattern. Lines 10 and 17 further specialize the pattern, specific to the two instructions, by placing constraints on the values of the fields defined in the instruction format. Lines 12 and 19 assist debugging by producing a disassembly format for the instructions in a printf-style declaration. Finally, lines 14 and 21 attach semantic behaviors to the instructions. Listing 2 shows the C-like DSL that describes the behaviors of the corresponding instructions. These descriptions are fed into an offline generator, which produces a fast instruction decoder, and a low-level IR emitter. The instruction decoder is used by the execution engine to decode guest 
basic blocks. The IR emitter is called for each decoded instruction, which produces a sequence of low-level IR instructions.

3.2.1. Native Code Production. Our execution engine compiles guest basic blocks at a time, but will extend to a trace-based approach if the branch targets are static and land on the same guest memory page. Guest basic blocks are terminated at page boundaries for memory protection purposes. Normal control flow out of a block is optimized utilising techniques from Spink et al. [2014], which includes directly chaining to other basic blocks that are part of the same memory page to avoid costly returns to the main execution loop. If a translation does not exist, or the destination does not live on the same page, then control is returned to the main execution loop, which will then handle the situation accordingly. We dedicate an x86 host register $(\% \mathrm{r} 15 \mathrm{~d})$ to tracking the guest PC, which significantly improves performance by avoiding an increment to a memory location (i.e., the emulated guest register file) on each instruction.

As each instruction is being translated to a sequence of IR instructions, we employ the partial evaluation technique described in Wagstaff et al. [2013] to constant-fold values known at compilation time into the IR. This technique also allows us to evaluate control flow within an instruction that can be resolved at compilation time, too, that is, control flow that depends on values that are constant.

After producing IR that represents the basic block being translated, we then perform some basic optimization passes (such as liveness analysis, control flow optimization, and dead code elimination) before performing a linear-scan register allocation pass. After allocating registers, we perform some final optimizations (such as register value re-use and dead code elimination) and then forward the IR to the instruction lowering pass that translates IR instructions into corresponding host instructions.

Although there are existing JIT compilers, we choose to implement our own JIT compiler for three main reasons as follows:

(1) It is not feasible to use compilers such as GCC and LLVM with our system, because they have dependencies on external libraries, such as libc, for example. The JIT compilation is performed inside the native VM, which is not running an operating system and does not have a C library.

(2) Compilers such as GCC and LLVM are too slow to be used as JIT compilers [Brandner et al. 2009], as they would introduce an unacceptable amount of compilation latency. Our JIT is specialized for fast dynamic binary translation, much like TCG in QEMU.

(3) Existing JIT compilers, for example, Tiny Code Generator (TCG) in QEMU, are not well suited for automatic retargeting from a high-level architecture description and would require additional modifications to support bare-metal code generation.

In Figure 4, Listing 3 shows an example ARM guest basic block that is encountered during the Linux kernel boot process. The IR emitter iterates over this block and, after an optimization pass, produces the IR shown in Listing 4. Finally, a quick templatebased lowering pass produces the native x 86 machine code shown in Listing 5 .

It can be seen here that multiple host basic blocks are produced from a single guest basic block. In this example, this occurs because of a predicated ARM instruction (movcs) that may or may not be executed, depending on the current state of the flags. Since we do not class predicated instructions as basic block terminators, additional control flow is required to account for this.

3.2.2. Exploitation of Hardware Features. Given that we are operating in a bare-metal environment, we have full control of an x 86 machine and so exploit our ability to use privileged instructions and access normally privileged features to generate efficient native code. Furthermore, we exploit our ability to switch the virtualized CPU into 
Listing 3: ARM guest basic block

\begin{tabular}{|lll|}
\hline ldr & r3, [r4] & // Load value from memory \\
cmp & r3, \#0x1000000 & // Compare value to constant \\
movcs & r0, \#1 & // Set ro to 1, if carry flag \\
bcs & $5412 \mathrm{dc}$ & $/ /$ Branch if carry flag \\
\hline
\end{tabular}

Listing 5: x86 host basic blocks

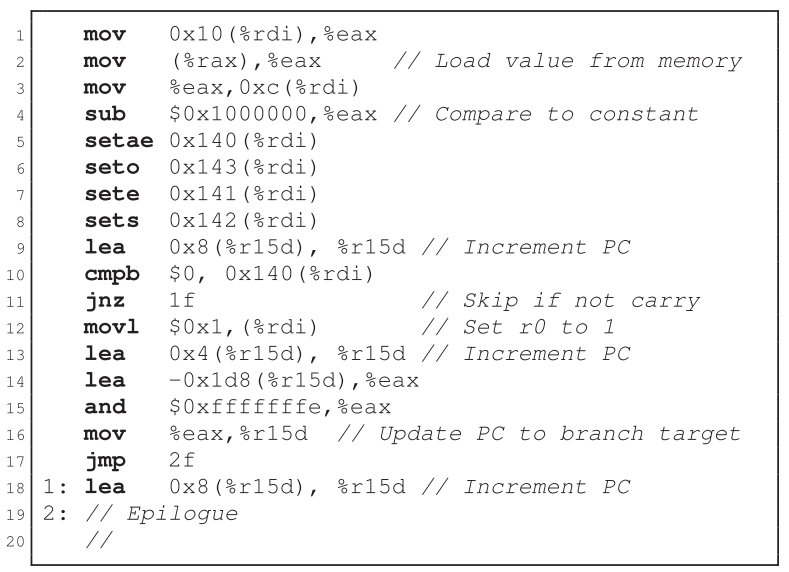

Listing 4: Execution Engine IR

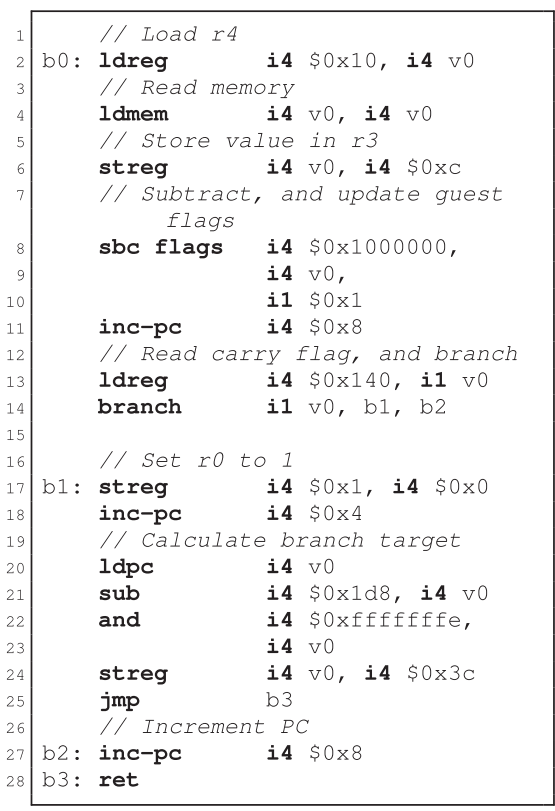

Fig. 4. Example inputs and outputs during the JIT compilation phase of CPU virtualization. ARM guest code is initially translated to an internal representation for optimization before $\mathrm{x} 86$ host code is generated and emitted.

and out-of-privileged mode (ring 0 in x86 terminology), so we can execute ARM guest code that usually runs in ARM user mode in x86 user mode and ARM guest code that usually runs in ARM system mode in x86 privileged mode. This enables us to utilise the user/kernel memory protection available in x86 page tables by mapping it to the corresponding ARM page table permissions. This is something that a user-space system like QEMU cannot do, as it is constrained by the host operating system.

We implement fast mode switching in x86 by using the syscall and sysret instructions, which provide efficient means of transitioning between user and privileged mode. We make use of the general-purpose segment register FS to point to a per-CPU data structure, which contains the state of the emulated ARM CPU and the GS segment register for efficient user-mode emulated memory accesses as described in Section 3.3.3.

We utilise the $\mathrm{x} 86$ call gate mechanism for invoking functions from user mode that require kernel mode permissions. This is an alternative to the slower software-interruptbased mechanism (i.e., using the int instruction).

3.2.3. Code Cache. In order to improve execution performance, translated guest basic blocks are kept in a code cache, indexed by physical address. The benefit of using physical addressing is that if the guest page tables are invalidated, we do not need to invalidate any compiled code. In fact, the only time we have to invalidate code is in the presence of self-modifying code or, more generally, when a page that has previously been executed is written to. We detect this occurrence by marking (physical) pages that have been executed with a flag and protecting those pages from being written to. When a memory fault occurs because of a write, and the page has been flagged, all cached code corresponding to that page is invalidated. If the memory fault was to an address on the page that was currently executing, then we return to the main execution loop via 
a non-local jump, since we cannot return to cached code that represents instructions that were potentially modified.

\subsection{MMU Virtualization}

One of the most important parts of full-system virtualization is the faithful emulation of the MMU, which, if implemented incorrectly, will lead to an unusable system and, if implemented poorly, can lead to severe performance penalties. Hardware extensions for same-architecture virtualization provide accelerated means of virtualizing the MMU of a guest machine on the host, but a problem arises when virtualizing a guest with a different architecture. As described in the motivating example (Section 1.1), the MMUs between two different architectures behave quite differently, and traditional full-system cross-architecture virtualization uses a (correct but slow) software MMU implementation to emulate this subsystem. Thus, much work has been done [Wang et al. 2015; Chang et al. 2014; Hong et al. 2015] in the area of software MMUs to reduce the translation penalty and hence increase overall throughput of the virtualization system.

Fundamentally, the function of the MMU is to translate a virtual address to a physical address, applying any permissions that may be defined for that access. Usually, this mapping is represented with page tables, with various levels of indirection to suit the granularity of the mapping. Full-system virtualization requires that every instruction that accesses virtual memory is subject to the behavior of the MMU. For the same-architecture case, memory instructions are mapped one to one, and the hardware extensions take care of performing the virtual-to-physical translation and permissions checking, but for cross-architecture virtualization, each memory access must be emulated in such a way as to perform the translation and permissions checking subject to the behavior of the guest platform.

Software approaches, when faced with a memory access (in the base case), will traverse the guest page table to resolve the physical address and check that the access satisfies the permissions imposed by the translation. These accesses will be subsequently sped up by introducing a software cache, much like a software TLB, so future memory accesses do not incur a penalty of a costly page table walk. When the guest page tables change, the software TLB will be flushed, and the process will start again.

Since the native VM is a bare-metal environment, we can take full control of the native MMU and use it to reflect the mappings of the guest, allowing us to use unmodified guest virtual addresses and to emulate the access with a single native instruction.

Definition 3.6 (Native MMU). The native MMU is the MMU that is part of the Native VM. In this example, the MMU is an x86-64 MMU, which has a four-level hierarchy.

Definition 3.7 (Native Page Table Entry). A native page table entry is an entry in the page table of the native MMU.

Definition 3.8 (Guest MMU). The guest MMU is a (software) implementation of the MMU that is part of the guest machine. In this example, it is an implementation of an ARMv7-A MMU. It is implemented as a service that takes a virtual address and returns either success (along with the corresponding physical address and a bitmask of allowed permissions) or failure (along with the type of failure).

Our approach to cross-architecture virtualization of the MMU is to present the lower 4GB (i.e., virtual addresses $0 \times 0$ to $0 x f f f f f f f f$ in the native VM's 48-bit address space) of virtual memory to the execution engine, as the $4 \mathrm{~GB}\left(2^{32}\right)$ of virtual memory required 


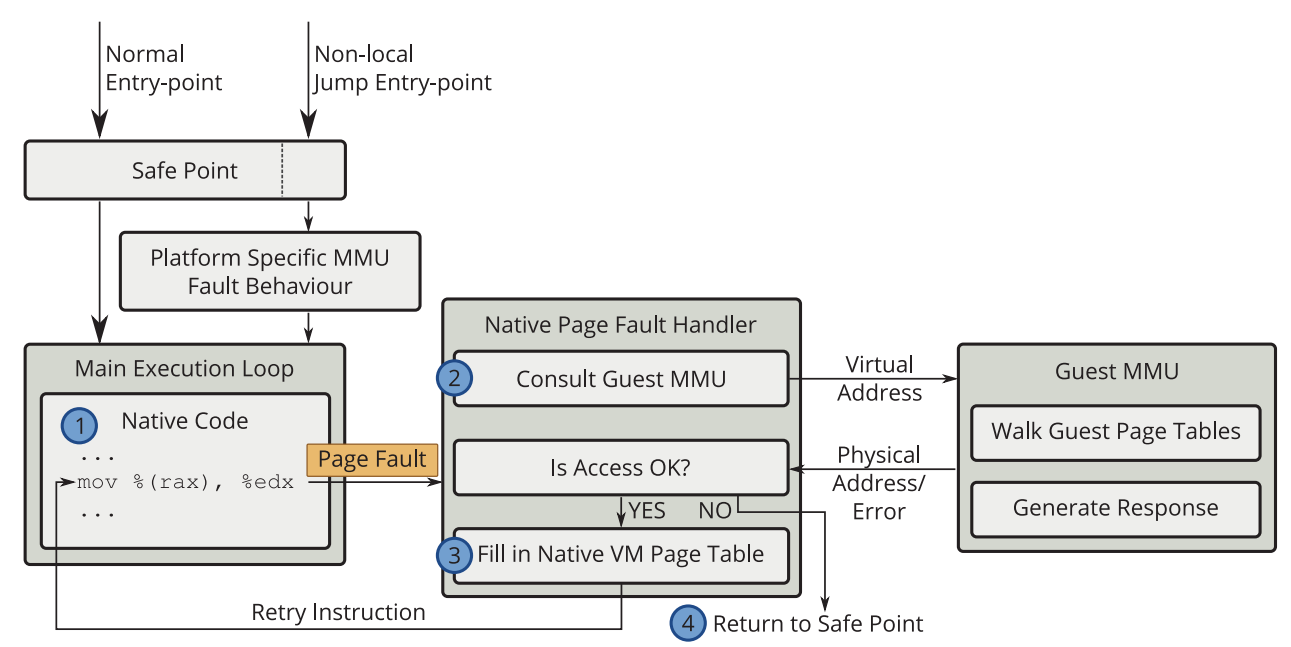

Fig. 5. Operation when virtualizing memory accesses. A memory access (1) is performed as a single native instruction, which, when accessing a virtual address for the first time, will cause a page fault in the Native VM. The native page fault handler will (2) consult the guest MMU implementation to determine if the mapping is valid and then either (3) fill in a native page table entry or (4) perform a non-local jump from the page fault handler back to a safe point to invoke platform specific memory fault handling.

for our 32-bit guest machine (see Figure 7). This area is now an exact 1:1 mapping of guest virtual addresses to native VM virtual addresses. Figure 5 shows how the various components work together. When we virtualize a memory access from the guest (whether a load, store, or fetch), we perform that access on the unmodified memory address directly, which will of course (for the 32-bit system we are virtualizing) lie in the lower 4GB region. The first time a memory address is accessed, it will cause a page fault inside the native VM, and at this point we consult the software implementation of the guest's MMU. The response is either the corresponding guest physical address or a fault condition. If the access is to be allowed, then we populate the $x 86$ page table of the native VM with an entry that maps the associated virtual page to the corresponding physical page of the guest and return to executing code. Further accesses to this page will now go via the native VM's page tables and hardware TLB.

To improve performance, when the guest MMU is asked for the translation, it also returns the allowed permissions associated with that mapping (such as read/write and user/privileged), so the native VM's page tables can be pre-populated with this information. This means that a read to a page that is also permitted to be written to will only fault once- the first time it is accessed.

On a 64-bit x86 machine, there are four levels of page tables, which we will refer to as L4 thru L1. The first entry in the (top-level) $\mathbf{L} 4$ page table represents the lower $0-512 \mathrm{~GB}$ of virtual memory, and we reserve this region to contain the entire $4 \mathrm{~GB}$ virtual address space of the guest, starting at virtual address $0 \times 0$. This means we can apply permission flags to the first entry in this table to control the entirety of the 4GB virtual address space. If the guest alters the content of their page tables, just as on actual hardware, then they are required to issue a TLB flush instruction, which we intercept and use as a signal to invalidate the native VM's page tables. As the native VM has a four-level page table, we deny access to the entire lower 4GB area of virtual memory, by clearing the page present flag in the first entry of the $\mathbf{L 4}$ page table, and then perform a native TLB flush. This makes invalidations very quick to perform. The next time a memory access happens, a page fault will occur, and the page tables will 


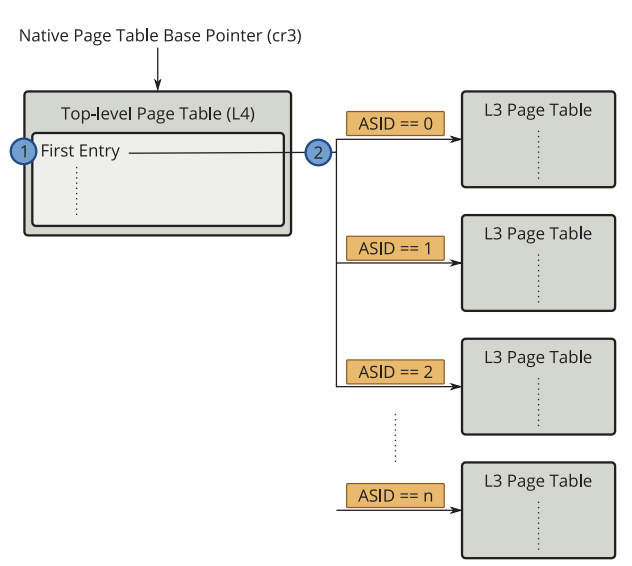

Fig. 6. The top-level (L4) page table remains static, and the pointer to the L3 page tables (1) are tracked with the ASID (2).

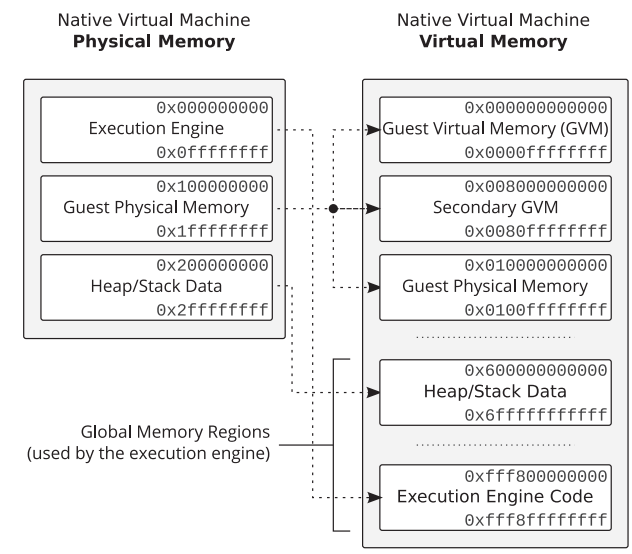

Fig. 7. Native VM Physical and Virtual memory organization. We reserve the bottom 512GB to contain the entire $4 \mathrm{~GB}$ virtual address space.

be rebuilt. This invalidation technique also applies when the guest changes the value of their own page table base pointer, which involves an implicit TLB flush.

An important corner-case to consider is the behavior of the system when an unaligned memory access spans a page boundary, for example, a 32-bit memory access to the last byte of a page. This situation is handled automatically by CAPTIVE, as the page faulting behavior between the guest and host systems is identical.

3.3.1. Address-space Identifier. Usually, changing the page table base pointer naturally causes a TLB invalidation, as the previous mappings are no longer valid. However, since the page table base pointer is changed on every context switch, this can lead to a severe performance penalty, especially in our virtualization environment when the native page tables need to be rebuilt each time. An approach to reduce this penalty is described by Wang et al. [2015] as "Private SPT," which utilizes the ARM ASID register to quickly switch between pre-populated mappings.

We take inspiration from this approach and use the ASID register to point to multiple L3 mappings, as shown in Figure 6. The top-level native page table (the L4 page table) remains static, but when the current ASID is changed by the guest, we replace the base pointer to the $\mathbf{L 3}$ page table (in the first slot of the $\mathbf{L 4}$ page table) and invalidate the native TLB. As previously described, the first entry in the $\mathbf{L} 4$ page table is used solely for the purpose of managing guest virtual memory, so even though it represents an address space $>4 \mathrm{~GB}$, it simplifies both our fast invalidation technique and changing the corresponding page tables that represent the guest $4 \mathrm{~GB}$ address space.

If this is the first time the ASID has been seen, then the normal page-fault lazy resolution process will occur as described previously, but if the ASID has already been encountered, then the page tables already contain mappings ready to be used (unless they were explicitly invalidated), without incurring any page faults.

We trap the special invalidation instructions issued by the guest to invalidate TLB entries by ASID and use these signals to invalidate the page tables that we have associated with that particular ASID.

This optimization only holds for guest platforms that have the concept of an ASID and guest kernels that actually use it (a limitation also encountered by Wang et al. [2015]). However, it is possible to extend this approach to track the guest platform's page table base pointer and maintain a set of mappings for "seen" page table bases. 
Listing 5: ARM input assembly

\section{Listing 6: CAPTIVE output assembly.}

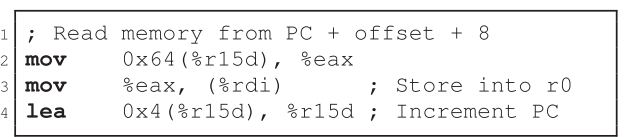

Listing 7: QEMU output assembly.

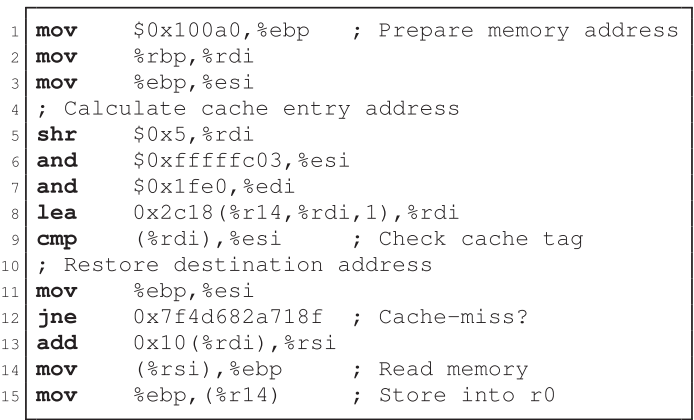

Fig. 8. An example of a PC-relative load instruction being translated by CAPTIVE and QEMU. CAPTIVE tracks the (virtual) PC in \% r15d and emits three instructions for this memory access, whereas QEMU emits 13 instructions that involve interrogating its address cache.

3.3.2. Native VM Memory Layout. As we are operating inside a native virtual machine, we have full control over the virtual machine's virtual memory, and so we exploit this opportunity for manipulating the virtual page mapping arbitrarily. We establish page mappings for the execution engine and heap/stack data areas and mark these entries as global, so they are not flushed from the TLB when the TLB is flushed. We provide a one-to-one mapping of guest physical memory in the virtual memory space so we can quickly access data by guest physical address. This is useful for the emulated MMU, as it uses physical address pointers to traverse the guest page tables.

3.3.3. Secondary Guest Virtual Memory. The secondary guest virtual memory mapping is part of an optimization for handling ARM ldrt and strt instructions, which perform memory accesses subject to user-mode memory permission checking while executing in kernel mode. These instructions are notoriously difficult to optimize [Ding et al. 2012], as they invoke behavior that must be specially handled. As they are defined, there is no direct mapping of this behavior from an ARM system to an x86-64 system; however, to maintain performance we employ a second region of guest virtual memory to optimize these accesses especially.

Since it is known at JIT compilation time that a particular memory access has these special semantics, we can emit an optimized mov instruction that offsets the calculated memory address against a base pointer held in the x86 GS register. This base pointer points to the base of the second virtual memory region, and so all memory accesses are made into this second region. Then, when a page fault occurs, we apply the appropriate semantics when faulting the page in. While this may sound like a guest architecturespecific optimization, it is implemented independent of the target architecture and so may be used (or not) by any platform that requires it.

3.3.4. Comparison to QEmU. QemU uses software-based MMU virtualization, and Listing 5 in Figure 8 shows an example ARM instruction that accesses memory from a PCrelative offset. This instruction loads a value from memory, residing at the address $P C+92+8$. Listing 7 shows the QEMU generated native code for this single instruction, which involves accessing a software cache, with a branch to a handler if a cache miss occurs. Our output code (shown in Listing 6 ) consists of performing the memory access directly on memory itself, using the unmodified value from the guest instruction and access permissions.

The other slight difference is the optimization performed for a PC-relative lookup. In QEMU's case, it can constant-fold the address of the memory access (0x100a0) into the 
generated assembly because it generates basic blocks for virtual pages. However, we generate basic blocks for physical pages, which may be accessed by any virtual address, and hence must read the PC register each time we wish to use it. As we map the guest PC to a host register, this improves code quality and adds virtually no performance penalty. This improvement in code quality is not because of an improvement in the quality of the JIT itself but rather because we have the ability to make memory accesses in this fashion.

\subsection{Device Virtualization}

In order to faithfully emulate a guest platform, we must also emulate the devices present on that platform, for example, timer devices, interrupt controllers, I/O devices, and so on. In order to do this, the hypervisor contains software emulations for the various devices that make up the platform. On a real guest platform, these devices are accessed by the guest through the memory subsystem; they are mapped into the physical memory space (and then mapped by the guest operating system into the virtual address space), and device registers are written to and read from with normal memory accesses. This approach to device communication increases flexibility (e.g., device accesses are subject to MMU translations and permissions checks) and reduces complexity for operating systems but adds a layer of complexity to virtualization frameworks wishing to emulate devices in a particular platform, as they must detect these accesses to device memory and handle them accordingly.

As our device implementation lives in the hypervisor (i.e., outside the native VM), memory accesses by the guest must be trapped back to the hypervisor, so they can be forwarded to the particular device being accessed. The most straightforward way to accomplish this with our infrastructure is to use the memory-mapped $I / O$ feature of KVM to intercept memory accesses to regions of guest physical memory that correspond to devices and handle them accordingly. This approach works well but suffers from a severe performance penalty, as every access to a device must perform a costly VM exit, and then the native guest instruction must be emulated by the hypervisor to fill in the data that was read or to extract the data that is to be written.

Device accesses in a full-system occur quite frequently. For example, a Linux system configured with a $100 \mathrm{~Hz}$ timer will be interrupted 100 times a second, and each interrupt requires the guest to interrogate the interrupt controller device to ascertain the cause of the interrupt, and then the timer device to read timing-related data, and then write to the devices to acknowledge and complete the interrupt.

Another approach is to make a hypercall using port-based I/O (PIO) instructions, which have slightly faster VM exit sequences, but this suffers from a fundamental problem: detecting a device access. As mentioned previously, a device access to a memory-mapped device is indistinguishable from a normal memory access at the instruction-level-it is performed with a normal memory access instruction (e.g., Idr in ARM). Therefore, we need a way to detect access to device memory and trap to the host using a faster hypercall mechanism. Since we are in control of the native VM MMU, and we know the locations of devices in physical memory (this is part of the platform configuration), we can simply mark any device page as inaccessible, so every memory access traps in the native VM rather than in the hypervisor.

Now that we are receiving a page fault in the native VM (which is faster than trapping to the hypervisor), there are two approaches to take:

(1) Translate the device access into a (slightly) faster PIO access, which still results in a VM exit, or

(2) use a message-passing implementation to communicate with the hypervisor, avoiding a VM exit. 


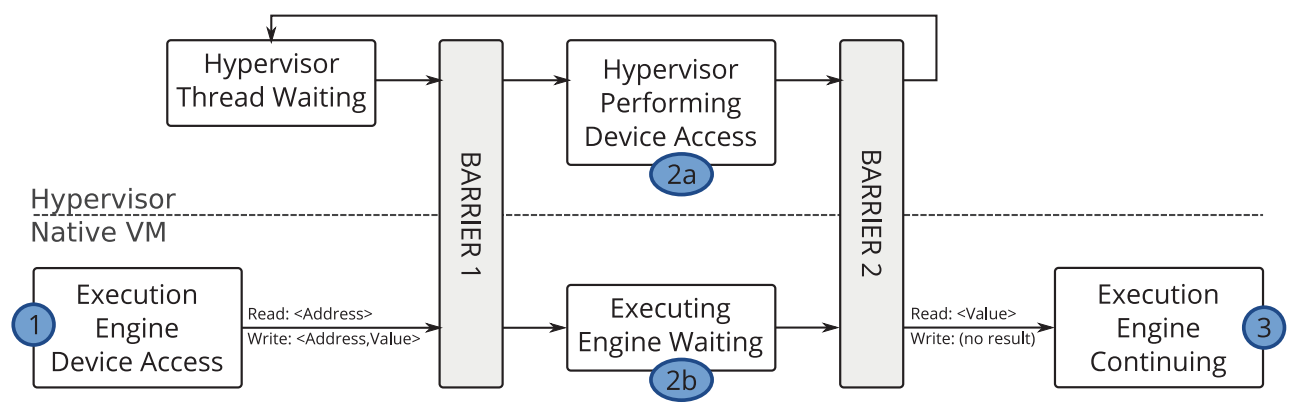

Fig. 9. An illustration of the fast device access operation, using synchronization barriers. When a device access is made (1), barrier 1 is entered by the guest (at which the host is already waiting), and the host performs the access on the emulated device (2a). Meanwhile, the guest waits for the host to complete the operation ( $2 \mathrm{~b})$. Then, when the access is complete, barrier 2 is entered by the host and execution by the guest continues (3).

We wish to avoid VM exits at all costs, as they introduce a significant amount of overhead [Ott 2009]. A VM exit with Intel VT and KVM requires storing the entire state of the virtual machine and performing a context switch back to user-space code. Returning to the VM (a VM entry) involves restoring this saved state.

For this reason, we implement (2), and once the native VM receives a page fault to a device memory page, we communicate with a hypervisor thread using a synchronization barrier system. This avoids a costly VM exit, as the virtualized CPU is simply spinning on a barrier, waiting for a response from the hypervisor. This sequence is shown in Figure 9 . When a device access is to be made Figure 9 (1), a data structure is prepared by the execution engine inside the native VM, and a synchronization barrier is entered. A hypervisor thread (which is already waiting on this barrier) resumes execution and deals with the device access request Figure 9 (2a). Meanwhile, the guest waits on a second barrier (Figure 9 (2b)) while the hypervisor is servicing the request, and, when the request is complete, the hypervisor writes the result back into the data structure and enters the barrier. This causes the execution engine to resume execution (Figure 9 (3)), extracting the necessary data from the request structure. The guest cannot proceed until the hypervisor has signalled that the data have been processed by the emulated device, and this is the reason for the second barrier.

3.4.1. Device Implementations. Unlike traditional same-architecture virtualization, where the possibility exists to para-virtualize hardware that exists on the host for use by the guest, or simply pass-through real hardware devices (e.g., using Intel VT-d), this same kind of mapping does not exist for cross-architecture virtualization, as it is unlikely that there are any one-to-one compatible devices available on the host system. Therefore, all guest platform devices are implemented in software, which faithfully emulate the behavior of the device they represent. An example of a device we implement in software is the ARM PrimeCell SP804, which is a two-channel timer device. This device is configured and interrogated by the guest through registers that are memory mapped. It is also capable of raising interrupts when a timeout occurs, depending on the mode of operation of the timer.

In the future, we hope to map similar devices (e.g., timer devices, etc.) to existing hardware devices. Even though their interfaces may be incompatible, it may be possible to configure the behavior of the devices in similar ways and avoid having to use full software implementations of the device.

3.4.2. Device Interrupts. Platform devices may raise interrupts to indicate that an event has occurred, such as a timer has timed out, or data are ready to be read. On a physical 


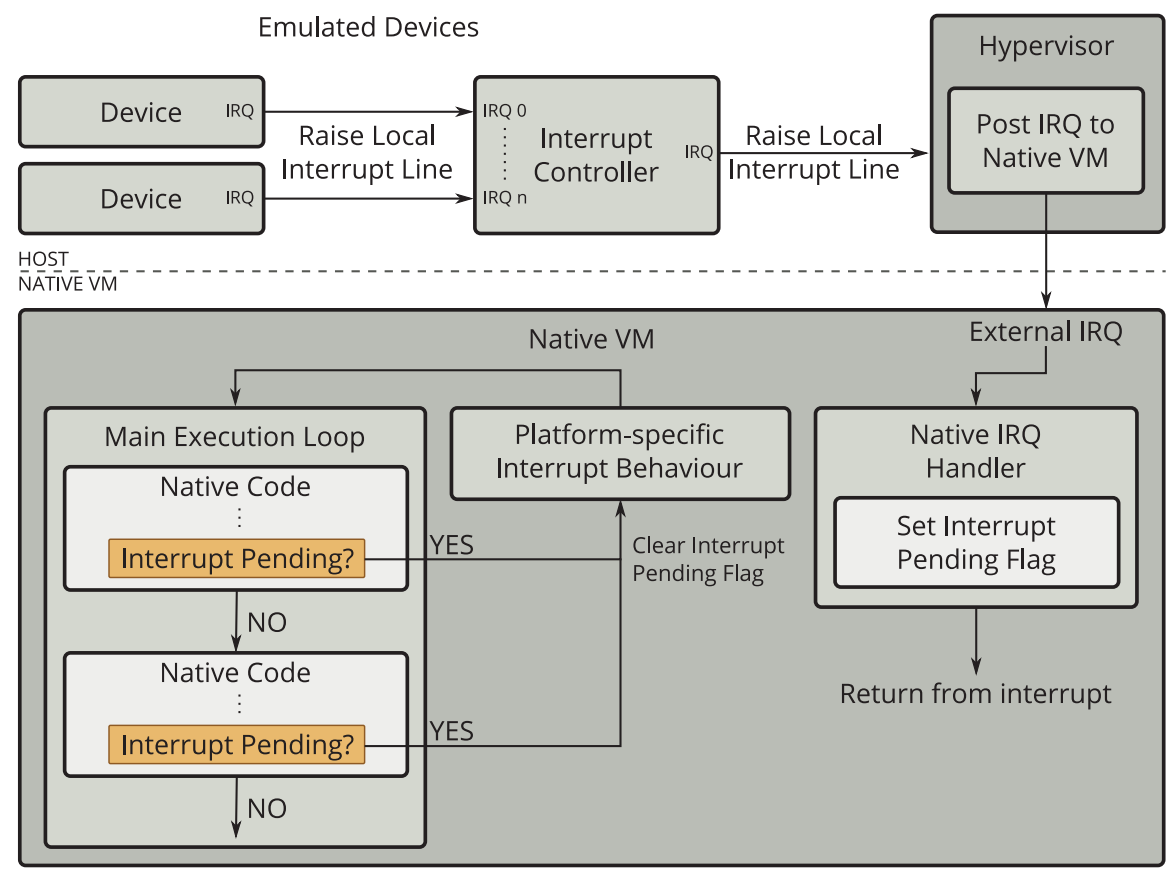

Fig. 10. An illustration of the injection of an IRQ into the native virtual machine to indicate that an emulated IRQ line has gone high.

platform, an interrupt controller would aggregate the individual interrupts from each device and trigger a physical interrupt line on the CPU to indicate that an interrupt has been raised. The CPU would enter its external interrupt handling routine and interrogate the interrupt controller to work out which device(s) raised the interrupt. The RealView Platform Baseboard Cortex-A8 [ARM 2011b] has such a setup with an ARM generic interrupt controller (GIC) that receives interrupts from devices and posts these to the CPU. We implement the GIC in software, but post real IRQs to the guest system, when the interrupt controller triggers a physical interrupt line on the CPU.

\subsection{IRQ Virtualization}

As described in the previous section, emulated devices may issue interrupts to the guest system by means of an interrupt controller. For the platform we are virtualizing, the interrupt controller is an ARM generic interrupt controller (GIC), which aggregates interrupts from other platform devices and presents them to the CPU.

Fundamentally, the CPU has a single physical interrupt line that is raised when an interrupt is pending and lowered when the interrupt is acknowledged. This interrupt line is toggled by our emulated GIC and is visible to the virtualized CPU. Figure 10 shows how on the rising edge of the interrupt line, we inject a native IRQ into the guest machine to inform it that the line has been raised. These interrupts of course happen asynchronously, for example, a timer device will run as a separate thread on the host machine, and, when its timeout occurs, it will trigger its own interrupt line, propagating through the interrupt controller and into the guest. The ideal situation would be to immediately invoke the platform-specific interrupt handling code, on the rising edge of the interrupt line, but this is not feasible for two reasons: 
Table I. Host Machine Description

\begin{tabular}{rlrl}
\hline System & Dell $^{\mathrm{TM}}$ PowerEdge $^{\mathrm{TM}}$ R610 & \\
\hline Architecture & $\mathrm{x} 86-64$ & Model & Intel $^{\mathrm{TM}} \mathrm{Xeon}^{\mathrm{TM}}$ E5-1620 \\
Cores/Threads & $4 / 8$ & Frequency & $3.50 \mathrm{GHz}$ \\
L1-Cache & $1 \times 4 \times 32 \mathrm{kB}(I \$ \& D \$)$ & L2-Cache & $1 \times 4 \times 256 \mathrm{kB}$ \\
L3-Cache & $1 \times 10 \mathrm{MB}$ & Memory & $16 \mathrm{~GB}$ \\
\hline
\end{tabular}

(1) The guest may not be running in native code (it may be handling a page fault) and,

(2) single-guest instructions are compiled to multiple-host instructions, which means the interrupt may happen part-way through the emulation of a guest instruction.

This is unacceptable, as guest instructions are not necessarily re-entrant and may have partially changed the state of the guest system mid-way through. Guest instructions need to appear to be atomic, and so they must have completed before we can divert to the interrupt handling behavior.

We solve this by setting an interrupt pending flag when in the native IRQ handler to indicate that the emulated interrupt line has gone high. This flag is checked by native code at the end of a guest basic block before it chains to the next. If the flag is set, then it is cleared and we leave native code to perform the guest platform behavior associated with servicing an interrupt. This defers asynchronous interrupt checking to basic block boundaries, which significantly improves performance over checking on instruction boundaries.

\section{EXPERIMENTAL EVALUATION}

In this section, we evaluate the performance of our system using industry standard benchmarks and compare CAPTIVE to the state-of-the-art cross-architecture virtualizer QEMU. We use the SPEC CPU2006 integer benchmark suite, as it is widely considered to be representative of real-world workloads. For our key results, we are using the reference input set, which requires a minor modification to the guest platform to increase the available guest physical memory for running the benchmarks. The amount of physical memory presented to the guest system is independent of the amount of physical memory available on the host system, as it is defined by the platform being emulated. We implement a RealView Platform Baseboard Cortex-A8 [ARM 2011b] platform, which specifies only 512MB of physical memory [ARM 2011a], but this is insufficient for running the reference input set of the benchmark suite. To overcome this limitation, we artifically increase the amount of physical memory in the guest platform to $2 \mathrm{~GB}$ in both CAPTIVE and QEMU, enabling the benchmark suite to run.

\subsection{Experimental Setup}

The platform that we are virtualizing is a RealView Platform Baseboard for Cortex-A8, which is fully supported by QEMU. We are running a vanilla ARM Linux 4.3.0 kernel, with the default configuration for the platform, except for the addition of a VirtIO block device to provide storage to the guest and an increase in physical memory as described previously. We are using an Arch Linux ARM user-space. The host machine is described in Table I.

\subsection{Key Results}

Our key results compare the performance of our system to QEMU version 2.4.0. Figure 11(a) shows the relative speedup of CAPTIVE compared to QEMU. In all cases, we outperform QEMU and, on average, by a factor of $2.5 \times$. Figure $11(\mathrm{~b})$ shows the absolute runtime of each benchmark in seconds. 


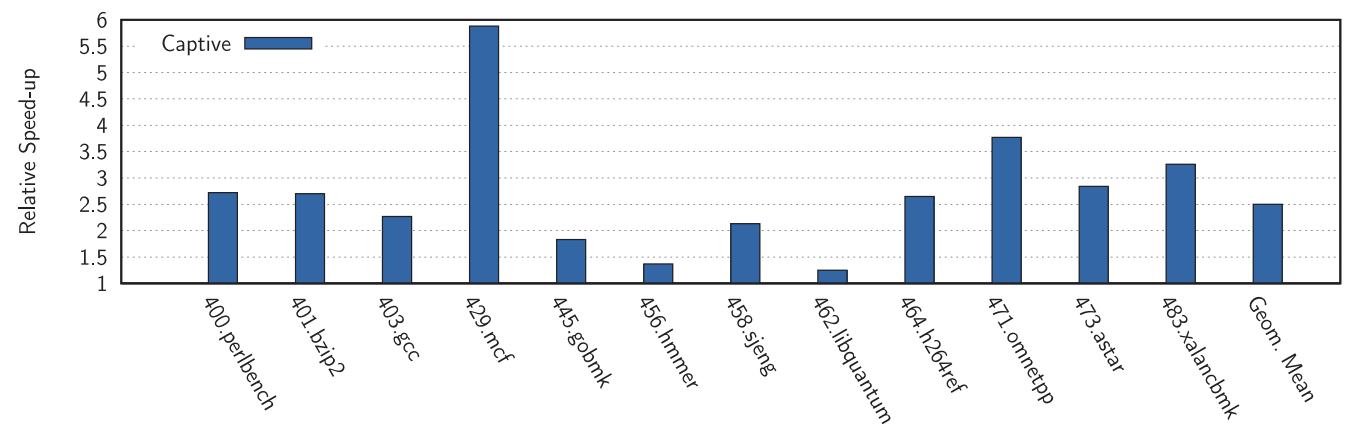

(a) Relative speed-up of the SPEC benchmark suite using the reference input set, in CAPTIVE over QEMUhigher is better.

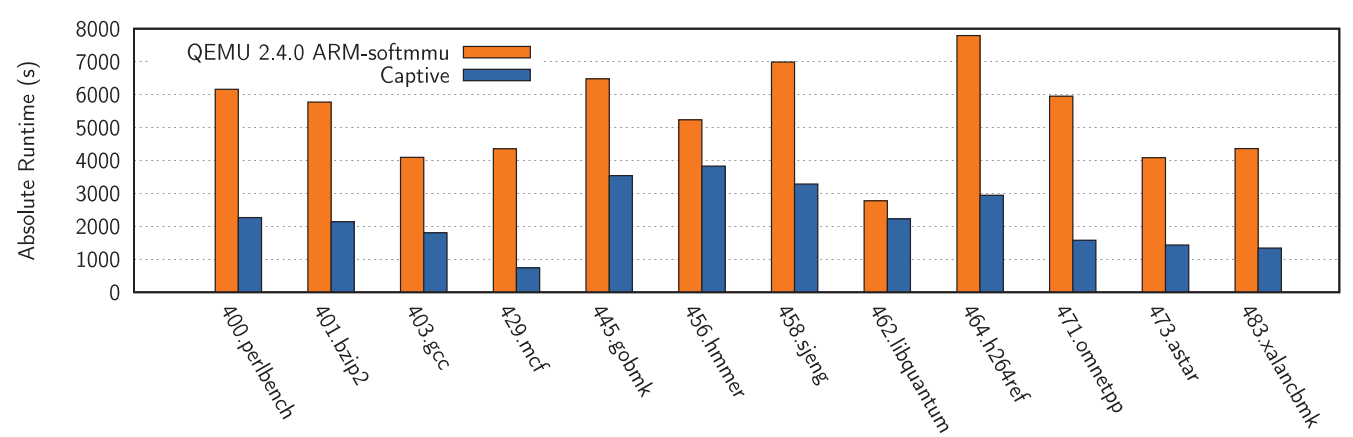

(b) Absolute runtime of the SPEC benchmark suite using the reference input set in seconds-lower is better.

Fig. 11. Key results: (a) shows relative speed-up, and (b) shows absolute runtime. On average, CAPTIVE is $2.5 \times$ faster than QEMU.

Of interest is 429. mcf, which gains a performance improvement of $5.88 \times$. This is due in part to the benchmark responding well to our optimizing DBT system, which produces highly optimal runtime code based on the dynamic behaviour of the benchmark versus the static optimization that is performed at compile time.

Only 2 of 12 benchmarks show speedups less than $1.5 \times$ yet still outperform the baseline QEMU. Given the acceptance of SPEC as a realistic workload, there are multiple characteristics that can affect simulation performance, and it is clear that the range of benchmarks exercise the simulation system in numerous ways, making it difficult to pinpoint any particular feature that causes fluctuations in performance.

\subsection{Comparison to Existing Techniques}

One of the most recent efforts to improve memory address translation performance in full-system simulators is in Wang et al. [2015] (herein referred to as HSPT), which describes a practical implementation of an embedded shadow page table, using Linux system calls (specifically mmap) to create an efficient guest-virtual to host-physical mapping similar to our own approach. In order to compare CAPTIVE to the HSPT implementation, we have extracted the published results from Wang et al. [2015] and implemented the same experimental setup by comparing the performance of CAPTIVE to the same version and configuration of the Android Emulator as used by HSPT. This enables us to make a relative performance comparison against the same baseline, even in the presence of different host machines.

Figure 12 shows that HSPT have achieved an average improvement of $1.94 \times$ (geometric mean) over the Android Emulator, using the Private Shadow Page Table (SPT), 


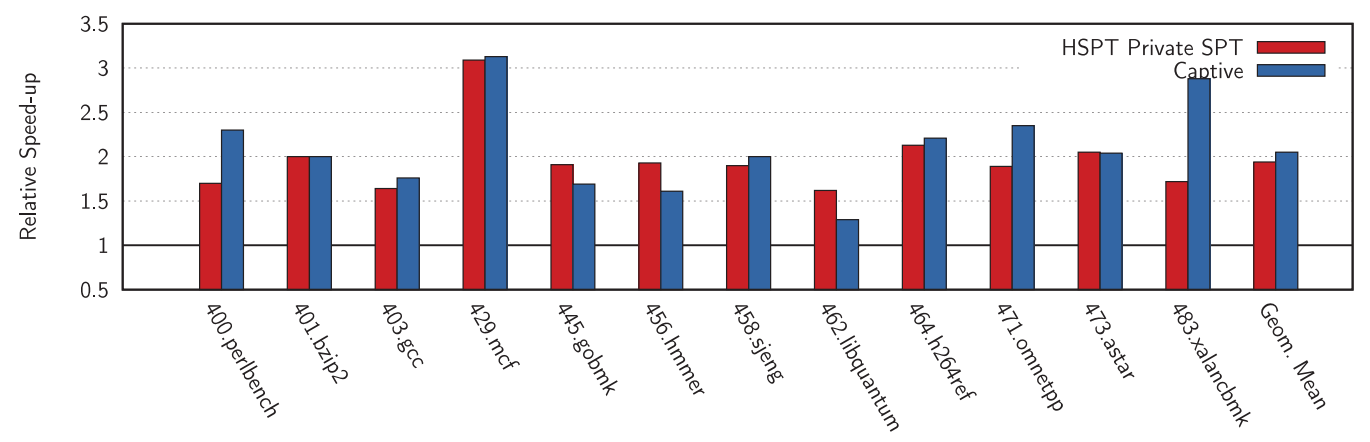

Fig. 12. Relative performance improvement of SPEC benchmarks by HSPT and CAPTIVE over the Android Emulator baseline; higher is better.

Table II. Absolute I/O Throughput for Various Configurations of Execution Environments; Cached Reads Are Subject to the Linux Kernel's Page Cache, and Buffered Reads Go Directly to the Real or Emulated Disk Device

\begin{tabular}{lll|rr}
\hline Hypervisor & Execution & Arch. & Cached & Buffered \\
\hline None & Native & x86 & $12384.21 \mathrm{MB} / \mathrm{s}$ & $173.52 \mathrm{MB} / \mathrm{s}$ \\
\hline VirtualBox & Intel-VT & $\mathrm{x} 86$ & $11941.43 \mathrm{MB} / \mathrm{s}$ & $91.64 \mathrm{MB} / \mathrm{s}$ \\
QEMU & KVM & $\mathrm{x} 86$ & $11881.06 \mathrm{MB} / \mathrm{s}$ & $102.72 \mathrm{MB} / \mathrm{s}$ \\
QEMU & DBT & $\mathrm{x} 86$ & $1265.03 \mathrm{MB} / \mathrm{s}$ & $79.80 \mathrm{MB} / \mathrm{s}$ \\
QEMU & DBT & $\mathrm{ARM}$ & $157.02 \mathrm{MB} / \mathrm{s}$ & $105.77 \mathrm{MB} / \mathrm{s}$ \\
\hline CAPTIVE & KVM/DBT & $\mathrm{ARM}$ & $1695.29 \mathrm{MB} / \mathrm{s}$ & $155.72 \mathrm{MB} / \mathrm{s}$ \\
\hline
\end{tabular}

whereas on average, CAPTIVE achieves a performance improvement of $2.05 \times$ (geometric mean).

In the majority of cases, we equal or surpass the speedup presented by HSPT; in particular, 483.xalancbmk in CAPTIVE shows a much greater speedup of $2.88 \times$ compared to $1.72 \times$ in HSPT. This is due in part to the I/O nature of this particular benchmark, and our optimized I/O and IRQ handling techniques give us a clear advantage here.

\subsection{I/O Performance}

In this section, we evaluate the performance of our $\mathrm{I} / \mathrm{O}$ virtualization using the standard Linux I/O performance measuring tool hdparm. We measure the I/O performance on a variety of virtualization configurations, including taking a measurement of the host system. We also introduce Oracle VirtualBox as another virtualization platform that uses Intel VT extensions and as such only supports same-architecture virtualization. For measurement of same-architecture virtualization I/O performance, VirtualBox and QEMU are given an x86 Linux distribution containing the hdparm tool. For cross-architecture virtualization, QEMU and CAPTIVE are provided with a file-system that exists as a normal file on the host machine's file-system. For QEMU/ARM and CAPTIVE/ARM, the platform device used to communicate this data back and forth is a VirtIO block device, which is fully supported by both hypervisors. VirtIO is a virtualization technology that enables efficient paravirtualization of various platform devices, such as network and disk devices. Our emulated disk is based on a VirtIO block device and is the only paravirtualized device in the platform.

Table II shows the absolute I/O throughput of the virtualization configurations, along with throughput on the native host platform, using two distinct metrics: cached and buffered.

Cached reads are subject to the Linux kernel page cache and as such represent the performance at which disk data can be accessed from the page cache in the guest 


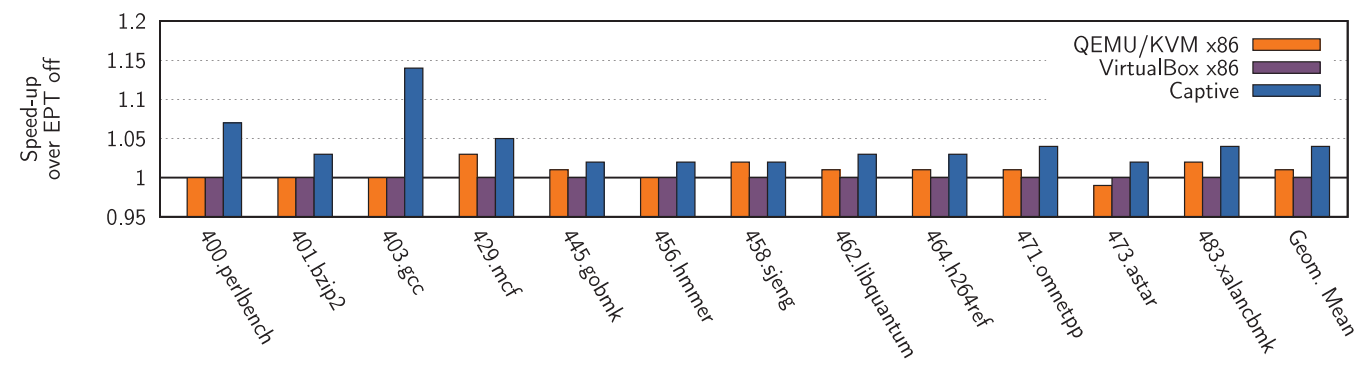

Fig. 13. Relative performance improvement gained by turning on Intel's EPT for the SPEC CPU2006 integer benchmark suite; higher is better. We show that the use of EPT has virtually no effect on the virtualization performance for the SPEC CPU2006 benchmark suite.

system. VirtualBox and QEmU/KVM make these accesses at virtually the same rate as the host platform, since there is no virtualization overhead for memory accesses. For QEMU/DBT, the accesses to this cache are subject to the software MMU implementation and therefore incur a significant access penalty. For QEMU/DBT, in the x86-on-x86 case, this causes a slowdown of $9.79 \times$ and a slowdown of $78.87 \times$ for the ARM-on-x 86 case. In CAPTIVE, the slowdown is only $7.3 \times$, improving over QEMU by $10.8 \times$.

Buffered reads indicate the rate at which data can be accessed directly from diskbypassing the page cache. For these experiments, host caching was disabled in each hypervisor, causing all accesses to the virtual disk device to go directly to the host file-system and then onto the underlying storage medium. All hypervisors suffer a slowdown over native for this case, as there will be overhead in accessing the virtual disk on the host file-system, but the slowdown over native for CAPTIVE is only $1.11 \times$ compared to QEMU/ARM, which is $1.64 \times$. Virtualization of the x86 guest machines on VirtualBox, QEMU/KVM, and QEMU/DBT all have even worse slowdowns, but this may be due to the implementation of the virtual disk device, which for these three hypervisors is an emulated IDE disk drive, as opposed to the para-virtualized VirtIO device used in QEMU/ARM and CAPTIVE.

\subsection{Additional Hardware Support for MMU Virtualization}

The latest version of Intel VT includes hardware support for accelerating virtualized guest page tables, which is branded as EPT. KVM can make full use of this technology, and this section evaluates the performance improvement of EPT over non-EPT backed virtualization. We use QEmU/KVM and Oracle VirtualBox (which fully supports EPT) to measure the impact of EPT on same-architecture virtualization. We have run six experiments to produce this data, three with EPT disabled in the respective hypervisor and three with EPT enabled. We then present the relative speedup of each hypervisor with EPT enabled over EPT disabled. For QEMU/KVM and Oracle VirtualBox, the experiments were naturally made on a virtualized x86-64 system, with $\mathrm{x} 86-64$ versions of the SPEC benchmark suite. For CAPTIVE, we have used the same setup as described in Section 4.1, with EPT enabled and disabled.

The data shows that, in our experiments, EPT does not make any significant improvement on the workloads we have tested. This is contrary to some published experiments, for example, VMware have conducted a performance evaluation of EPT in VMware [2009], which shows that EPT can improve performance of MMU-intensive benchmarks by $48 \%$ and MMU microbenchmarks by up to $600 \%$. However, our measurement of the impact of EPT on the SPEC CPU2006 benchmarks shows that the performance increase is negligible, which is also the conclusion drawn by Buell et al. [2013] and Merrifield and Taheri [2016]. Figure 13 shows the relative performance 


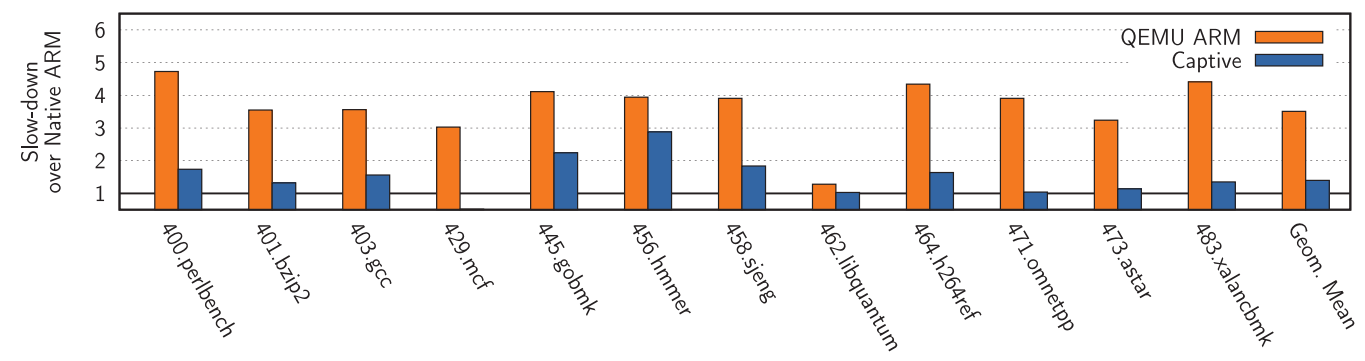

Fig. 14. Relative slowdown of QEMU and CAPTIVE over native execution on a physical ARM platform (ODROID-XU using Samsung Exynos5422 Cortex-A15 2.0GHz quad-core and Cortex-A7 quad-core CPUs); lower is better. On average, CAPTIVE is $1.4 \times$ slower than the hardware platform compared to a $3.51 \times$ slowdown for QEMU.

improvement of the SPEC benchmark suite, running on both a virtualized x86 system (using Qemu/KVM and Oracle VirtualBox) and on a virtualized ARMv7-A system (using our virtualization hypervisor). On average, there is virtually no improvement for QEMU and VirtualBox and only 3\% for CAPTIVE.

\subsection{Slowdown Over Native Execution on High-End Hardware}

We have evaluated the performance of CAPTIVE, compared to execution of the benchmarks on an ARM hardware platform. We have collected runtimes on an ODROID-XU, and Figure 14 shows the relative slowdown of both CAPTIVE and QEmu. On average, CAPTIVE is $1.4 \times$ slower than native execution of SPEC on an ARM platform, whereas QEMU is 3.51× slower. Again, of interest is the 429.mcf benchmark that actually shows a speedup over native. This is again partly due to the JIT compiler discovering optimizations that can be made dynamically but also due to larger CPU cache (e.g., L1) sizes on the host platform.

\section{RELATED WORK}

Instruction-set simulation is an active field of research, and a large number of techniques for the efficient implementation of either user mode or full system simulators has been published, for example, Böhm et al. [2011], Böhm et al. [2010], Witchel and Rosenblum [1996], Binkert et al. [2011], Patel et al. [2011], Sandberg et al. [2015], Yourst [2007], Bellard [2005], Ding et al. [2011], Magnusson et al. [2002], Qin and Malik [2003], and AMD Developer Central [2010]. In Table III, we provide an overview of well-known simulators and their capabilities and implementation techniques.

Early work in the context of Simics introduced a software caching mechanism that improved the performance of interpreted memory operations by reducing the number of calls to complex memory simulation code [Magnusson and Werner 1994]. More recently, Chang et al. [2014], Wang et al. [2015], and Hong et al. [2015] have presented novel schemes for speeding up address translation in full-system simulators. These two schemes are the closest matches to our work documented in the literature. In Chang et al. [2014], a shadow page table — called embedded shadow page table (ESPT)—is embedded into the address space of a cross-ISA DBT system. Similarly to CAPTIVE, ESPT uses the hardware memory management unit in the CPU to translate memory addresses, instead of software translation. However, the original ESPT approach has a few drawbacks. For example, its implementation relies on a loadable kernel module (LKM) to manage the shadow page table. Using LKMs is less desirable for system virtual machines due to portability, security, and maintainability concerns. Hence, a different implementation - called HSPT-adopts a shared memory mapping scheme to maintain the shadow page table using only mmap system calls [Wang et al. 2015]. In 
Table III. Comparison of Simulators: Techniques and Capabilities

\begin{tabular}{l|cccccc}
\hline Simulator & Engine & $\begin{array}{c}\text { Full- } \\
\text { System }\end{array}$ & $\begin{array}{c}\text { Multi- } \\
\text { Core }\end{array}$ & Detailed & $\begin{array}{c}\text { Hardware } \\
\text { Accelerated }\end{array}$ & Target ISA \\
\hline ArcSim & Parallel DBT & Yes & Yes & Config. & No & User Retargetable \\
Embra & DBT & Yes & Yes & Cache & No & MIPS R3000/R4000 \\
gem5 & Discr. Event & Yes & Yes & Yes & No & User Retargetable \\
MARSS & DBT & Yes & Yes & Yes & No & Intel x86 \\
OVPSim & DBT & Yes & Yes & No & No & Multiple available \\
pFSA & Direct Exec. & Yes & No & Sampling & For same ISA & Intel x86 \\
PTLsim & Virtualization & Yes & No & Yes & No & Intel x86-64 \\
QEMU & DBT & Yes & Yes & No & No & Multiple available \\
QEMU & KVM & Yes & Yes & No & For same ISA & Multiple available \\
PQEMU & DBT & Yes & Yes & No & No & ARM11MPCore \\
Simics & Interpreter & Yes & Yes & Approx. & No & Multiple available \\
Simit-ARM & DBT & Yes & No & No & No & ARM v5 \\
SimNow & DBT & Yes & Yes & (COTSon) & No & Intel x86, AMD64 \\
\hline CAPTIVE & DBT & Yes & Yes* & Cache* & Yes & User Retargetable \\
\hline
\end{tabular}

(*) Multi-core virtualization and optional cache modelling are beyond the scope of this article.

Section 4.3, we show a side-by-side performance comparison between this improved HSPT scheme and the approach taken by CAPTIVE. Dynamic resizing of a software TLB is proposed in Hong et al. [2015]. Using per-page-table utilization information the size of the software TLB is adjusted for each process separately.

\section{SUMMARY, CONCLUSION, AND FUTURE WORK}

We have introduced new techniques for cross-architecture virtualization using hardware-accelerated processor extensions and implemented these ideas in a hypervisor called CAPTIVE. The key contribution is the mapping of guest-system MMU behavior to host-system MMU behavior, and we improve over the state-of-the-art simulator QEMU on average $2.5 \times$. We show that CAPTIVE is better than existing techniques to improve MMU virtualization. There are three major routes that we wish to take to extend our work:

(1) We wish to extend the capability of our system to 64-bit guests and find efficient ways of exploiting our existing infrastructure to handle the larger address space.

(2) We wish to implement a multicore version of the execution engine to support platforms with multiple (possibly heterogeneous) processor cores.

(3) We wish to explore the possibility of mapping guest platform devices to real host devices, for example, timer devices, eliminating hypervisor emulation overhead.

\section{REFERENCES}

AMD Developer Central. 2010. AMD SimNow simulator. Retrieved from http://developer.amd.com/toolsand-sdks/cpu-development/simnow-simulator/.

ARM. 2011a. About the PB-A8. (2011). Retrieved from http://infocenter.arm.com/help/topic/com.arm.doc. dui0417d/BABCHBFC.html\#CHDFGCFB Retrieved 02-June-2016.

ARM. 2011b. RealView Platform Baseboard for Cortex-A8 User Guide. Retrieved from http://infocenter.arm. com/help/index.jsp?topic=/com.arm.doc.dui0417d/index.html.

Rodolfo Azevedo, Sandro Rigo, Marcus Bartholomeu, Guido Araujo, Cristiano Araujo, and Edna Barros. 2005. The ArchC architecture description language and tools. Int. J. Parallel Program. 33, 5 (Oct. 2005), 453-484. DOI : http://dx.doi.org/10.1007/s10766-005-7301-0

Fabrice Bellard. 2005. QEMU, a fast and portable dynamic translator. In Proceedings of the Annual Conference on USENIX Annual Technical Conference(ATEC'05). USENIX Association, Berkeley, CA, 41-41. 
Nathan Binkert, Bradford Beckmann, Gabriel Black, Steven K. Reinhardt, Ali Saidi, Arkaprava Basu, Joel Hestness, Derek R. Hower, Tushar Krishna, Somayeh Sardashti, Rathijit Sen, Korey Sewell, Muhammad Shoaib, Nilay Vaish, Mark D. Hill, and David A. Wood. 2011. The gem5 simulator. SIGARCH Comput. Archit. News 39, 2 (Aug. 2011), 1-7. DOI : http://dx.doi.org/10.1145/2024716.2024718

Igor Böhm, Tobias J. K. Edler von Koch, Stephen C. Kyle, Björn Franke, and Nigel Topham. 2011. Generalized just-in-time trace compilation using a parallel task farm in a dynamic binary translator. In Proceedings of the 32Nd ACM SIGPLAN Conference on Programming Language Design and Implementation (PLDI'11). ACM, New York, NY, 74-85. DOI : http://dx.doi.org/10.1145/1993498.1993508

Igor Böhm, Björn Franke, and Nigel P. Topham. 2010. Cycle-accurate performance modelling in an ultrafast just-in-time dynamic binary translation instruction set simulator. In Proceedings of the 2010 International Conference on Embedded Computer Systems: Architectures, Modeling and Simulation (ICSAMOS 2010), Fadi J. Kurdahi and Jarmo Takala (Eds.). IEEE, 1-10. DOI:http://dx.doi.org/10.1109/ ICSAMOS.2010.5642102

Florian Brandner, Andreas Fellnhofer, Andreas Krall, and David Riegler. 2009. Fast and accurate simulation using the LLVM compiler framework. In Proceedings of the 1st Workshop on Rapid Simulation and Performance Evaluation: Methods and Tools (RAPIDO).

Jeffrey Buell, Daniel Hecht, Jin Heo, Kalyan Saladi, and H. Reza Taheri. 2013. Methodology for Performance Analysis of VMware vSphere under Tier-1 Applications. VMware technical journal. Retrieved from https://labs.vmware.com/vmtj/methodology-for-performance-analysis-of-vmware-vsphereunder-tier-1-applications.

Jianjiang Ceng, Weihua Sheng, Jeronimo Castrillon, Anastasia Stulova, Rainer Leupers, Gerd Ascheid, and Heinrich Meyr. 2009. A high-level virtual platform for early MPSoC software development. In Proceedings of the 7th IEEE/ACM International Conference on Hardware / Software Codesign and System Synthesis (CODES+ISSS'09). ACM, New York, NY, 11-20. DOI : http://dx.doi.org/10.1145/1629435.1629438

Chao-Jui Chang, Jan-Jan Wu, Wei-Chung Hsu, Pangfeng Liu, and Pen-Chung Yew. 2014. Efficient memory virtualization for cross-ISA system mode emulation. In Proceedings of the 10th ACM SIGPLAN/SIGOPS International Conference on Virtual Execution Environments (VEE'14). ACM, New York, NY, 117-128. DOI : http://dx.doi.org/10.1145/2576195.2576201

J. H. Ding, P. C. Chang, W. C. Hsu, and Y. C. Chung. 2011. PQEMU: A parallel system emulator based on QEMU. In Proceedings of the 2011 IEEE 17th International Conference on Parallel and Distributed Systems (ICPADS). 276-283. DOI : http://dx.doi.org/10.1109/ICPADS.2011.102

Jiun-Hung Ding, Chang-Jung Lin, Ping-Hao Chang, Chieh-Hao Tsang, Wei-Chung Hsu, and Yeh-Ching Chung. 2012. ARMvisor: System virtualization for ARM. In Proceedings of the Ottawa Linux Symposium.

K. Ebcioglu, E. Altman, M. Gschwind, and S. Sathaye. 2001. Dynamic binary translation and optimization. IEEE Trans. Comput. 50, 6 (Jun. 2001), 529-548. DOI : http://dx.doi.org/10.1109/12.931892

Adam Gerber and Clifton Craig. 2015. Learn Android Studio: Build Android Apps Quickly and Effectively (1st ed.). Apress, Berkely, CA.

Apala Guha, Kim hazelwood, and Mary Lou Soffa. 2010. DBT path selection for holistic memory efficiency and performance. In Proceedings of the 6th ACM SIGPLAN/SIGOPS International Conference on Virtual Execution Environments (VEE'10). ACM, New York, NY, 145-156. DOI : http://dx.doi.org/ 10.1145/1735997.1736018

Ding-Yong Hong, Chun-Chen Hsu, Cheng-Yi Chou, Wei-Chung Hsu, Pangfeng Liu, and Jan-Jan Wu. 2015. Optimizing control transfer and memory virtualization in full system emulators. ACM Trans. Archit. Code Optim. 12, 4, Article 47 (Dec. 2015), 24 pages. DOI : http://dx.doi.org/10.1145/2837027

Intel. 2016. Intel Virtualization Technology (Intel VT). Retrieved from http://www.intel.com/content/www/ us/en/virtualization/virtualization-technology/intel-virtualization-technology.html Retrieved 26-April2016.

Daniel Jones and Nigel Topham. 2009. High speed CPU simulation using LTU dynamic binary translation. In Proceedings of the 4th International Conference on High Performance Embedded Architectures and Compilers (HiPEAC'09). Springer-Verlag, Berlin, 50-64. DOI : http://dx.doi.org/10.1007/978-3-540-92990-1_6

Naveen Kumar, Bruce R. Childers, Daniel Williams, Jack W. Davidson, and Mary Lou Soffa. 2005. Compiletime planning for overhead reduction in software dynamic translators. Int. J. Parallel Program. 33, 2 (June 2005), 103-114. DOI : http://dx.doi.org/10.1007/s10766-005-3573-7

KVM. 2016. KVM. Retrieved from http://www.linux-kvm.org/page/Main_Page.

Peter S. Magnusson, Magnus Christensson, Jesper Eskilson, Daniel Forsgren, Gustav Hållberg, Johan Högberg, Fredrik Larsson, Andreas Moestedt, and Bengt Werner. 2002. Simics: A full system simulation platform. Computer 32, 2 (Feb. 2002), 50-58. Retrieved from http://dlib.computer.org/co/books/co2002/ pdf/r2050.pdf. 
Peter S. Magnusson and Bengt Werner. 1994. Some Efficient Techniques for Simulating Memory. Technical Report R94. Swedish Institute of Computer Science technical report.

Dirk Merkel. 2014. Docker: Lightweight linux containers for consistent development and deployment. Linux J. 2014, 239, Article 2 (March 2014).

Timothy Merrifield and H. Reza Taheri. 2016. Performance implications of extended page tables on virtualized x86 processors. In Proceedings of the 12th ACM SIGPLAN/SIGOPS International Conference on Virtual Execution Environments (VEE'16). ACM, New York, NY, 25-35. DOI:http://dx.doi.org/ $10.1145 / 2892242.2892258$

David Ott. 2009. Virtualization and Performance: Understanding VM Exits. Retrieved from https://software. intel.com/en-us/blogs/2009/06/25/virtualization-and-performance-understanding-vm-exits.

A. Patel, F. Afram, S. Chen, and K. Ghose. 2011. MARSS: A full system simulator for multicore x86 CPUs. In Proceedings of the 2011 48th ACM / EDAC/IEEE Design Automation Conference (DAC'11). 1050-1055.

Wei Qin and S. Malik. 2003. Flexible and formal modeling of microprocessors with application to retargetable simulation. In Proceedings of the Design, Automation and Test in Europe Conference and Exhibition. 556-561. DOI : http://dx.doi.org/10.1109/DATE.2003.1253667

A. Sandberg, N. Nikoleris, T. E. Carlson, E. Hagersten, S. Kaxiras, and D. Black-Schaffer. 2015. Full speed ahead: Detailed architectural simulation at near-native speed. In Proceedings of the 2015 IEEE International Symposium on Workload Characterization (IISWC). 183-192. DOI :http://dx.doi.org/10.1109/ IISWC.2015.29

Tom Spink, Harry Wagstaff, Björn Franke, and Nigel Topham. 2014. Efficient code generation in a regionbased dynamic binary translator. In Proceedings of the 2014 SIGPLAN/SIGBED Conference on Languages, Compilers and Tools for Embedded Systems. ACM, 3-12.

David Ung and Cristina Cifuentes. 2000. Machine-adaptable dynamic binary translation. In Proceedings of the ACM SIGPLAN Workshop on Dynamic and Adaptive Compilation and Optimization (DYNAMO'00). ACM, New York, NY, 41-51. DOI : http://dx.doi.org/10.1145/351397.351414

VMware. 2009. Performance Evaluation of Intel EPT Hardware Assist. Technical Report. VMware. Retrieved from https://www.vmware.com/pdf/Perf_ESX_Intel-EPT-eval.pdf.

Harry Wagstaff, Miles Gould, Björn Franke, and Nigel Topham. 2013. Early partial evaluation in a JITcompiled, retargetable instruction set simulator generated from a high-level architecture description. In Proceedings of the Annual Design Automation Conference (DAC'13). ACM, New York, NY, Article 21, 6 pages. DOI : http://dx.doi.org/10.1145/2463209.2488760

Zhe Wang, Jianjun Li, Chenggang Wu, Dongyan Yang, Zhenjiang Wang, Wei-Chung Hsu, Bin Li, and Yong Guan. 2015. HSPT: Practical implementation and efficient management of embedded shadow page tables for cross-ISA system virtual machines. In Proceedings of the 11th ACM SIGPLAN/SIGOPS International Conference on Virtual Execution Environments. ACM, 53-64.

Emmett Witchel and Mendel Rosenblum. 1996. Embra: Fast and flexible machine simulation. In Proceedings of the 1996 ACM SIGMETRICS International Conference on Measurement and Modeling of Computer Systems (SIGMETRICS'96). ACM, New York, NY, 68-79. DOI : http://dx.doi.org/10.1145/233013.233025

M. T. Yourst. 2007. PTLsim: A cycle accurate full system x86-64 microarchitectural simulator. In Proceedings of the IEEE International Symposium on Performance Analysis of Systems Software (ISPASS'07). 23-34. DOI : http://dx.doi.org/10.1109/ISPASS.2007.363733

Received May 2016; revised August 2016; accepted September 2016 